\title{
GLOBAL RECESSIONS AS A CASCADE PHENOMENON WITH INTERACTING AGENTS
}

\author{
Paul Ormerod ${ }^{*}$ and Amy Heineike, Volterra Consulting, London \\ 135c Sheen Lane \\ London SW14 8AE \\ UK
}

\section{December 2008}

* corresponding author

pormerod@,volterra.co.uk; aheineike@,volterra.co.uk

We are grateful to an anonymous referee for some extremely helpful comments.

JEL: $\quad$ C69, E32, N10

Keywords: cascades; recessions; international transmission; interacting agents

\begin{abstract}
This paper examines global recessions as a cascade phenomenon. In other words, how recessions arising within one or more countries might percolate across a network of connected economies. An agent based model is set up in which the agents are Western economies. A country has a probability of entering recession in any given year and one of emerging from it the next.

In addition, the agents have a threshold propensity, which varies across time, to import a recession from the agents most closely connected to them. The agents are connected on a network, and an agent's neighbours at any time are either in (state 1) or out (state 0) of recession. If the weighted sum exceeds the threshold, the agent also goes into recession.

Annual real GDP growth for 17 Western countries 1871-2006 is used as the data set. The model is able to replicate three key features of the statistical distribution of recessions: the distribution of the number of countries in recession in any given year, the duration of recessions within the individual countries, and the distribution of 'wait time' between recessions i.e. the number of years between them.

The network structure is important for the interacting agents to replicate the stylised facts. The country-specific probabilities of entering and emerging from recession by themselves give results which are by no means as well matched to the actual data
\end{abstract}




\section{Introduction}

There is a very large literature within economics on business cycles within individual economies, going back at least as far as the work of Marx in the $19^{\text {th }}$ century. It is fair to say that no genuine consensus has emerged within the profession of either the causes of cycles in general or recessions in particular. For example, the monumental work of Friedman and Schwartz (1963) offers a monetary explanation of the Great Depression in the United States. But despite the fact that this was published nearly half a century ago, its findings are still a matter of dispute. Dow, for example, a senior economist at the OECD and the Bank of England, describes them as 'unsatisfactory and implausible' (2000).

The major work of Burns and Mitchell (1946) illustrates how difficult the task is of forming a satisfactory theory which is able to explain different recessions even within the same country. They offer a very detailed description of all the business cycles in America to that, and a striking feature of their descriptions is how the recessions differ. For example, they suggest that the period of recessions has a range of between two and twelve years.

But it is not the purpose of this paper to give an account of how recessions might arise purely within an individual country. Rather, the aim of this paper is to examine global recessions as a cascade phenomenon. In other words, how recessions arising within one or more countries might percolate across a network of connected economies.

The last decade or so has seen considerable interest on information cascades and herding behaviour within economics (for example, Banerjee (1992) and Bikchandani et.al. (1992) are two seminal papers within the discipline). This paper examines the evidence for whether recessions arising within individual countries have a tendency to spread across countries.

Several examples come to mind of how recessions might spread in this way. In 1932, for example, Keynes described how recessions could be spread by non-co-operative policies: 'Each nation, in an effort to improve its relative position, takes measures injurious to the absolute prosperity of its neighbors; and, since its example is not confined to itself, it suffers more from similar action by its neighbors than it gains by such action itself. Practically all the remedies popularly advocated to-day are of this internecine character. Competitive wage reductions, competitive tariffs, competitive liquidation of foreign assets, competitive currency deflations, competitive economy campaigns - all are of this beggar-my-neighbor description.'

Empirical evidence for a different mechanism is provided by, Eichengreen et.al. (1996). They use a panel of quarterly data for 20 industrial countries for the period 1959-1993 to 
show that currency crises and associated losses of real output spread more easily between countries which are closely tied by international trade linkages. Less formally, recessions might spread through the international percolation of expectations, of which the current sub-prime lending crisis is a potential example. Foster and Fleith (2002), for example, consider a nonlinear model in which agents exchange their opinions and information with each other. Their model yields multiple equilibria, or attracting distributions, that are persistent but subject to sudden large jumps

The above list is not meant to be exhaustive, nor is it our intention to offer a description of the exact mechanism by which recessions might cascade across countries. Rather, we consider evidence over a long time period of the distribution of the number of leading Western economies in recession in any given year, and examine the extent to which this can be explained by a model based on the principles of cascades.

Section 2 describes the data and the key empirical phenomena we require the model to be able to replicate. Section 3 sets out the theoretical model, and section 4 discusses the results.

\section{The data and the stylised facts}

We use a long run of data from the main capitalist economies, going back to the late $19^{\text {th }}$ century when they can all be plausibly be regarded as being essentially industrial rather than agricultural in nature

The definition of a recession is the conventional one of a year in which the rate of growth of real GDP in a country is less than zero. Bordo and Helbing (2003), for example, discuss the advantages and disadvantages of NBER-based definitions of recessions, which consider a wider range of indicators. However, as they note, the two approaches give very similar results in terms of the timing of recessions. Further, and decisive in terms of the choice of definition of recession in this context, NBER-type business cycle dates do not exist for all the countries over the 1870-2006 period.

There are of course issues of data accuracy with data over such a long span of time. Romer $(1994,1999)$ for example subjects the NBER data for the United States to careful analysis. She suggests, for example, that what may appear to be lower volatility in the post-World War Two period compared to the pre-war data may be due to better data collections. She does argue that, even taking this into account, recessions have become less frequent. Whether this opinion survives the next decade is, given current circumstances, very much a moot point.

The most carefully constructed data set across the capitalist economies is that of Maddison (1995), and annual real GDP data for 17 countries 1870-1994 is taken from this source. The 1995-2006 data is from the IMF database. Strictly speaking, the two sources are not exactly comparable since the Maddison data is in real Geary-Khamis dollars and the IMF in real domestic currency, but given that we are working with annual GDP growth, this is of little consequence. 
The countries ${ }^{1}$ are: Australia, Austria, Belgium, Canada, Denmark, Finland, France, Germany, Italy, Japan, Netherlands, New Zealand, Norway, Sweden, Switzerland, United Kingdom and United States.

In terms of the validation of the model and the calibration of its parameters, three stylised facts are selected:

- the statistical distribution of the length of recessions within individual countries

- the statistical distribution of the number of years between recessions within individual countries

- the statistical distribution for the number of countries in recession in any one year

It is not the purpose of this paper to attempt to characterise these empirical distributions by a known theoretical distribution, but to see if the data generated by the model is consistent with the empirical distributions.

There is in fact a small literature on the distribution of the length of recessions within individual countries (for example, Ormerod and Mounfield (2001), Gaffeo et.al. (2003), Ausloos et.al.(2004), Wright (2005)), but the ability of formal analysis to discriminate between different hypotheses is limited by the very small number of data points.

In total, there are 255 instances of recessions in the 17 capitalist economies over the 1871-2006 period. Of these, around two-thirds, 164 to be exact, lasted only one year and 58 lasted 2 years. Of the remainder, in 20 cases a country was in recession for three successive years, in 6 cases for 4 years, in 5 for 5 years, and there is one example each of recessions lasting 6 and 7 years respectively.

Table 1 summarises this information

Table 1 Distribution of duration of recessions, 17 capitalist countries, 18712006

$\begin{array}{llllllll}\text { Duration: years } & 1 & 2 & 3 & 4 & 5 & 6 & 7 \\ & 164 & 58 & 20 & 6 & 5 & 1 & 1\end{array}$

The data is perhaps slightly more stretched than a simple exponential (Laherrere and Sornette (1998)), but the latter gives a good fit to the data (e.g. Wright, op.cit.) Ormerod (2009) confirms this point, but suggests that a Weibull with shape parameter less than one is superior. However, to re-iterate, our purpose is to see if the theoretical model generates results which are compatible with this data, not to enter into the debate on the nature of the distribution..

\footnotetext{
${ }^{1}$ In the Maddison data set, Swiss GDP data is only available but only from 1900 on an annual basis, and the data for Japan from 1885
} 
In terms of the distribution of the number of years between recessions, there are unavoidable problems with the data, relating to truncation. For each country, the years prior to the first recorded recession in the data set are ignored, as are the years to 2006 at the opposite end of the time period. So, for example, the last recorded recession in no fewer than 7 countries was in 1993, so for them the period 1993-2006 is ignored in calculating the 'wait times' between recessions. For recessions of longer duration than a single year, the period between recessions is counted as one year for the second and each subsequent year.

\section{Figure 1 here}

Gaffeo et.al (op.cit.) suggest that the statistical distribution of recoveries - the number of years between recessions - is different from that of recessions. A power law appears to hold for recoveries, but only after restricting the sample by getting rid of shorter episodes. Redelico et.al. (2008) argue more firmly that the distribution of the number of years between recessions in Latin American countries follows a power law. Both these studies, however, essentially rely on log-log ordinary least square regression analysis. Such regressions can lead to biased estimates of the power law exponent (Goldstein et.al., 2004), and alternative estimation methods should be used (Newman, 2005)

.In terms of the number of countries in recession in any one year, there does not appear to be an existing literature which attempts to characterise its statistical distribution. The data shows that in no fewer than 40 out of the 136 years, no single country was in recession, and there is no example of a year in which all 17 were simultaneously in recession. The largest single number in any one year is 15 in 1931, closely followed by 14 in 1930. Of the 17 countries, 12 were in recession in 1914 and 1932, and 11 in 1917, 1918 and 1975.

The histogram of the number of countries in recession in any one year is set out below in Figure 2.

\section{Figure 2 here}

\section{The cascade model}

The model is populated by 17 countries, the sizes of which correspond to the size of the actual economies in dollar terms in 1955. The year 1955 is chosen because it is close to the mid-point of the sample period but is not subject to the distortions of either the Great Depression of the 1930s or of World War Two and its aftermath.

The model proceeds in a series of steps. In the first step, each country goes into recession with probability $\pi$. The mechanism by which an individual country enters recession of its own accord, as it were, is not specified, but the key feature is that it is stochastic, a feature, for example, of real business cycle theory. Once in recession, the country remains so until it emerges from the recession according a further stochastic process: in 
step 2 of the solution, those which were in recession in step 1 recover with probability $\rho$. The remainder enter recession with probability $\pi$.

The model proceeds over a total of 136 steps, the length of the sample period 1871-2006.

Each economy is connected to a particular number of neighbours and recessions are propagated across the connections. Each is assigned a threshold chosen at random from a uniform distribution on $[\tau, 1]$. The neighbours are assigned a value of 0 if not in recession and 1 if they are in recession. The economy goes into recession if the weighted sum of the neighbours in the period is greater than $\tau$, where the weights are the sizes of the economies. So there is heterogeneity amongst the time-steps in terms of the propensity to 'import' recession at any given point in time.

There are therefore two sources of recession, the stochastic element $\pi$ which is particular to each individual time step, and the propagation mechanism across the network of economies.

\section{The network structure}

Evidence on the nature of the relevant topology of the network of connections is sparse. One possible source of transmission is of course the world trade network, and a small literature exists here, but the results are mixed. Serrano and Boguna (2003), for example, suggest that the world trade network is approximated by a small world topology. Fagiolo et.al. (2008) find that the distribution of weighted trade links is similar to a power law, suggesting that a scale free network is more appropriate.

The investigations with the model were carried out with both a small world and a scalefree network. For comparison, a random network was also investigated.

Small world networks (Watts and Strogatz (1998)) consist of a network of vertices whose topology is that of a regular lattice, with the addition of a low density $\mu$ of connections between randomly-chosen pairs of vertices. This latter can be either an addition to the connections on a regular lattice or can replace the relevant number of such connections. A scale-free network is a network whose degree distribution follows a power law, at least asymptotically. That is, the fraction $P(k)$ of nodes in the network having $k$ connections to other nodes is distributed for large values of $k$ as $P(k) \sim k^{-\gamma}$. (A valuable introduction to recent socio-economic uses of network theory is Durrett (2006)).

The distinction between the two is by no means clear cut, as Amaral et.al. (2000) pointed out. Both, for example, have clustering coefficients much larger than those of random networks, and the diameter of the network, quantified by the average shortest distance between two vertices, increases logarithmically with the number of vertices $n$.

Empirically, given the small number of nodes in this network ( the number of countries, 17) there are substantial similarities between the three kinds of graph. To illustrate the point, Table 2 shows the average path length and the mean degree (the average number of 
neighbours each node has) for the optimal parameters selected after extensive searches using each type of network, both for the network and for the other parameters in the model.

Table 2 Path length and mean degree of best examples of each network

Path length Mean degree

Random

Small world

Scale free
2.36

1.73

1.97
3.16

2.24

3.65

In all three cases, very satisfactory results were obtained, and the model-generated data was similar to the empirical distributions of all three of the stylised facts. The best results with a small world topology are marginally superior to those with a scale-free or a random network, so these are the ones reported here. Full details of the extensive parameter searches carried out with all three networks are available from the authors.

\section{$5 \quad$ Model calibration and results}

There are five parameters to choose in calibrating the model:

$\pi$ : the probability of a country going into recession in any given year of its own accord

$\rho: \quad$ the probability of a country spontaneously recovering form recession in any given year

$\tau$ : $\quad$ the minimum threshold indicating the propensity to import a recession

$\mathrm{k}$ : the number of immediate neighbours of a country

$\mu$ : the probability of a connection between any randomly-chosen pairs of countries

The word 'neighbour', to emphasise, does not mean geographic neighbour, but the countries which are closest to any given country in terms of their influence on whether or not it goes into recession.

We established initially that the results are essentially robust with respect to the choice of the re-wiring parameter, $\mu$. The graph theoretic properties of the small world network used in the model show why. The average path length is the average number of steps along the shortest paths for all possible pairs of network vertices. A short average path length facilitates the transfer of information and/or influence.

Figure 3 plots the average path length of the network for different values of $\mathrm{k}$ with $\mu$ set equal to $0.05,0.10$ and 0.15 . The path length is, certainly for $\mathrm{k}>1$, essentially invariant to the choice of $\mu$. Preliminary investigation of the properties of the model confirmed 
that they are robust to the choice of $\mu$, certainly for any reasonable values, and we therefore held $\mu$ fixed at 0.1 across all combinations of parameters subsequently investigated.

\section{Figure 3 here}

We do not know at any point in time which countries exercise the most influence on any other. We therefore obtain 500 separate solutions of the model, each over 136 steps. In each solution, the exact structure of the small world network is drawn afresh, with parameters $\mathrm{k}$ and $\mu$.

We carried out a complete grid search over the four parameters, with $\mathrm{k}=1,2,3$ and $\pi, \rho$ and $\tau$ varying in steps of 0.1 in the interval $[0,1]$. This gave 3992 separate variants of the model, each of which was solved 500 times, each over 136 steps.

We selected according to the following criteria. For each combination of parameters and for each of the 500 solutions, Kolmogorov-Smirnov tests were carried out on three separate null hypotheses that the statistical distribution of the model generated data and the actual data are the same for:

- the number of countries in recession at any point in time

- $\quad$ the duration of recessions within individual countries

- $\quad$ the 'wait time' between recessions in individual countries

The Anderson-Darling test is known to have higher power than the Kolmogorov-Smirnov test (for example, Stephens (1974)). However, there are two main reasons for choosing the latter. The Matlab manual, for example, states that "the asymptotic P-value [of the $\mathrm{K}-\mathrm{S}$ test] becomes very accurate for large sample sizes, and is believed to be reasonably accurate for sample sizes $\mathrm{N} 1$ and $\mathrm{N} 2$ such that $(\mathrm{N} 1 * \mathrm{~N} 2) /(\mathrm{N} 1+\mathrm{N} 2) \geq 4$ '. In the current context, each individual calculation of the test compares two sets of data where $\mathrm{N} 1=\mathrm{N} 2$ $=136$, so this criteria is very easily satisfied. Second, the K-S test is distribution free in the sense that the critical values do not depend on the specific distribution being tested. The A-D test makes use of the specific distribution in calculating critical values.

Across the 500 solutions of each combination of the parameters, we averaged the pvalues at which each of the null hypotheses were rejected.

For around 93 per cent of the parameter combinations investigated, at least one of the null hypotheses was rejected at a mean p-value across the 500 solutions of less than 0.05 . These could be discarded. The rejection of the vast majority of the combinations, incidentally, offers evidence that with the sample size of 136, the K-S test is of sufficient power to discriminate. 
With the remaining 7 per cent of the parameter combinations, we re-ran the model to obtain 5,000 solutions in each case, and selected the parameters for which the sum of the p-values is maximised.

Figures 4(a)-(c) plot the sum of the p-values across the initial sets of 500 solutions for the parameters $\pi, \rho$ and $\tau$.

\section{Figures 4a to c here}

The parameters for which the average sum of the p-values across 5,000 solutions is maximised are $\pi=0.1, \rho=0.7, \tau=0.5$ and $\mathrm{k}=3$.

The mean p-value across 5,000 solutions in the Kolmogorov-Smirnov test of the hypothesis that the distribution of the number of countries in recession in any given year in the model is the same as the actual data is 0.868; the mean p-value in the KolmogorovSmirnov test of the hypothesis that the distribution of the duration of recessions in the model, is the same as the data is 0.929; the mean p-value in the Kolmogorov-Smirnov test of the hypothesis that the distribution of wait time between recessions in the model, for wait time up to and including 31 years is the same as the data is 0.635 .

Pooling the 5,000 solutions, each run over 136 time steps, gives a total of 680,000 observations. The model does produce a small percentage of periods (2.42) when there is a truly global recession in which all 17 countries are in recession in the same year.

In terms of the duration of recessions generated by the model, 99.88 per cent of all recessions are between 1 and 7 years, the range of the actual data. The very large number of observations $(1,409,798$ recessions) generated by the model leads to a longer 'tail' in terms of duration than in the actual data. The longest is in fact 15 years, although only 0.02 per cent of recessions are longer than 10 years.

The proportion of recessions by duration is very similar in the model output and the actual data.

$$
1 \quad 2 \quad 3 \begin{array}{ccccc}
\text { Duration } & 4 & 5 & 6 & 7
\end{array}
$$

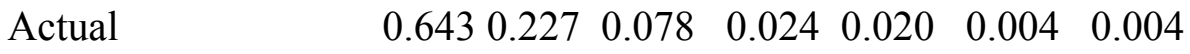

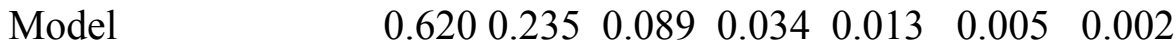

The very large number of runs of the model generate wait times of up to 88 years with the parameters above. Initial experiment showed that even longer wait times could be generated with different combinations of the parameters. Very long tails - given that 99 per cent of actual wait times are between 1 and 31 years and the longest of all is 46 years - appeared to discriminate on the p-value of the Kolmogorov-Smirnov test against 
models with such tails, and so in the selection criteria the test was carried out comparing model and actual wait times of between 1 and 31 years. So over the bulk of the data, the model accurately replicates the data.

This simple model is therefore able to replicate three key empirical features of recessions.

It is useful to examine the role which the network plays in generating these results. The network was removed by setting $\mu=\mathrm{k}=0$. Again, a grid search was carried out over different combinations of $\pi$ and $\rho$. The optimal values in this context were $\pi=0.15$ and $\rho=0.55$.

The approximations to the data, across 5000 solutions of the model, were not unreasonable. On formal statistical tests the average p-value at which the null hypotheses that the distributions of model and actual data are the same are $0.265,0.944$ and 0.748 . The latter are comparable to the model including the network, but the former, relating to the number of countries in recession, is distinctly lower.

Further, qualitatively, the comparison with the actual data is not as good as with the model including the transmissions network. For example, 64.3 per cent of actual recession last for only a single year. As noted above, in the network model 62.0 per cent do so, but with the no-network model the percentage is 55.3. For wait times, 38.2 per cent of actual wait times are just 1 year, with the network model 39.5 per cent and the no-network model 46.3 per cent. As Figure 1 shows, the distribution of the number of countries in recession in any given year is slightly erratic, but in the actual data 58.1 per cent of all observations have the value 0,1 or 2 . With the network model, this percentage is 53.4, and the no-network model 26.3.

So the network is important in terms of obtaining a more accurate correspondence of the model results with the data. Choosing appropriate values for the domestic probabilities of entering or recovering from a recession generates a reasonable approximation to two of the three empirical features which the model is intended to capture, but adding the network definitely improves the model.

\section{Conclusion}

Using a data base of annual real GDP growth 1871-2006 in 17 Western economies, we obtain the empirical distributions of three key features of economic recessions. The number of countries in recession in any given year, the duration of recessions within individual countries and the wait times between recessions in individual countries.

A model of interacting agents in is able to replicate these three key facts. The agents are countries, which have a probability of both entering and emerging from recession. In addition, they are connected on a small world topology. The neighbours of any given agent are assigned the value 1 if they are in recession and 0 if not. If the weighted sum of neighbours exceeds a random, heterogeneous threshold chosen for that time step, the 
agent also enters recession. The role of the network is important in the ability of the model to replicate the key facts on recession.

Of course, the fact that a model is able to replicate key facts does not mean that it is the only possible model which can do so. There is always, potentially, an infinite number of possible alternative models, although none as yet exist in the literature. However, the model offered here is distinctly parsimonious, using only four parameters. Alternative models need to be able to either explain the key facts selected here with a smaller number of parameters, or if a greater number is used, to be able to account for more stylised facts on recessions. As it stands, the simple model of this paper provides evidence that international transmission across countries is a feature of recessions under capitalism.

\section{References}

L.A.N. Amaral, A.Scala, M.Barthélémy and H.E.Stanley, 'Classes of small world networks', Proceedings of the National Academy of Science, 11149-52, 2000

M. Ausloos, J. Miskiewicz and M. Sanglier, The Durations of Recession and Prosperity: Does their Distribution follow a Power or an Exponential law?' Physica A,339, 548-558, 2004

A.V. Banerjee, 'A Simple Model of Herd Behaviour', Quarterly Journal of Economics, 107, 797-817, 1992

S. Bikchandani, J. Hirshleifer and I. Welch, 'A Theory of Fads, Fashion, Custom and Cultural Change as Informational Cascades', Journal of Political Economy, 100, 9921026, 1992

M Bordo and T Helbing, 'Have National Business Cycles Become More Synchronised?', National Bureau of Economic Research Working Paper 10130, NBER, Cambridge, MA, 2003

A.F. Burns and W.C.Mitchell, Measuring Business Cycles, New York, NBER, 1946

C.R.Dow, Major Recessions: Britain and the World 1920-1995, Oxford University Press, 2000

R. Durrett, Random Graph Dynamics, Cambridge University Press, 2006

B. Eichengreen, A. Rose and C. Wyplosz, 'Contagious Currency Crises', National Bureau of Economic Research Working Paper 5681, 1996

G.Fagiolo, J,Reyes, S.Schiavo, 'The World-Trade Web: Topological Properties, Dynamics and Evolution', arXiv:0807.4433v1 [physics.soc-ph], 2008

J. Foster and B. Flieth, 'Interactive Expectations', Journal of Evolutionary Economics, 12, 375-396, 2002

M.Friedman and A.Schwartz, A Monetary History of the United States 1867-1960, Princeton University Press for the NBER, 1963

E.Gaffeo, M.Gallegati, G.Giulioni and A.Palestrini, 'Power Laws and Macroeconomic Fluctuations', Physica A, 324, 408-416, 2003

M.L.Goldstein, S.A.Morris and G.C.Yen, 'Problems with Fitting to the Power-Law Distribution', European Physical Journal B, 41, 255-258, 2004

J.M. Keynes, 'The World's Economic Outlook', Atlantic Monthly, 149, 521-526, 1932 
J. Laherrere and D Sornette, 'Stretched Exponential Distributions in Nature and Economy: 'Fat tails' with Characteristic Scales', European Physical Journals, B2, 525539,1998

A. Maddison, Monitoring the World Economy, OECD, Paris, 1995

M.E.J.Newman, 'Power Laws, Pareto Distributions and Zipf's Law', arXiv/0412004v2, 2005

P.Ormerod, 'Recessions and the Resilience of the Capitalist Economies', Risk Management, forthcoming, 2009

P.Ormerod and C.Mounfield, 'Power Law Distribution of Duration and Magnitude of Recessions in Capitalist Economies: Breakdown of Scaling', Physica A, 293, 573-582, 2001

F.O.Redelico, A.N.Proto and M.Ausloos, 'Power Law for the Duration of Recession and Prosperity in Latin American Countries', Physica A, 387, 6330-6336, 2008

C.D.Romer, ' Remeasuring Business Cycles', Journal of Economic History, 54, 573-609, 1994

C.D.Romer, 'Changes in Business Cycles: Evidence and Explanations', Journal of Economic Perspectives, 13, 23-44, 1999

M.A. Serrano and M. Boguna, 'Topology of the World Trade Web', Physical Review E68, 015101(R), 2003

M.A.Stephens, 'EDF Statistics for Goodness of Fit and Some Comparisons', Journal of the American Statistical Association, 69, 730-737, 1974

D.J.Watts and S.H. Strogatz, 'Collective Dynamics of "Small-World" Networks', Nature, 393, 440-442, 1998

I.Wright, 'The Distribution of Recessions Follows an Exponential and not a Power Law', Physica A, 345, 608-610, 2005 
Number of years between recessions in 17 countries 1871-2006

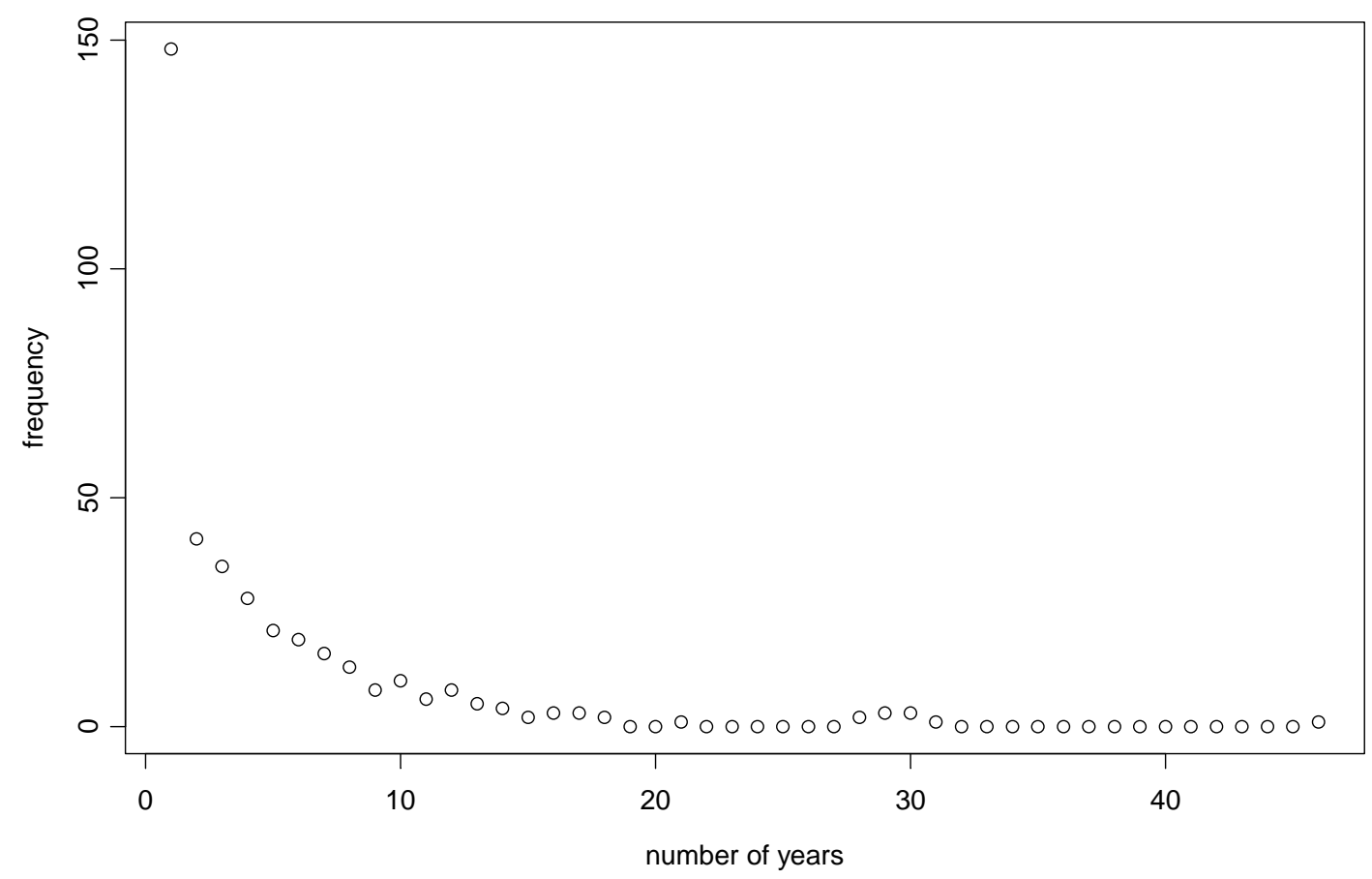

Figure 1 Distribution of number of years between recessions, 17 Western economies, 1871-2006; truncated data. The most frequent observation is 1 year i.e. observed when recessions last more than a single year

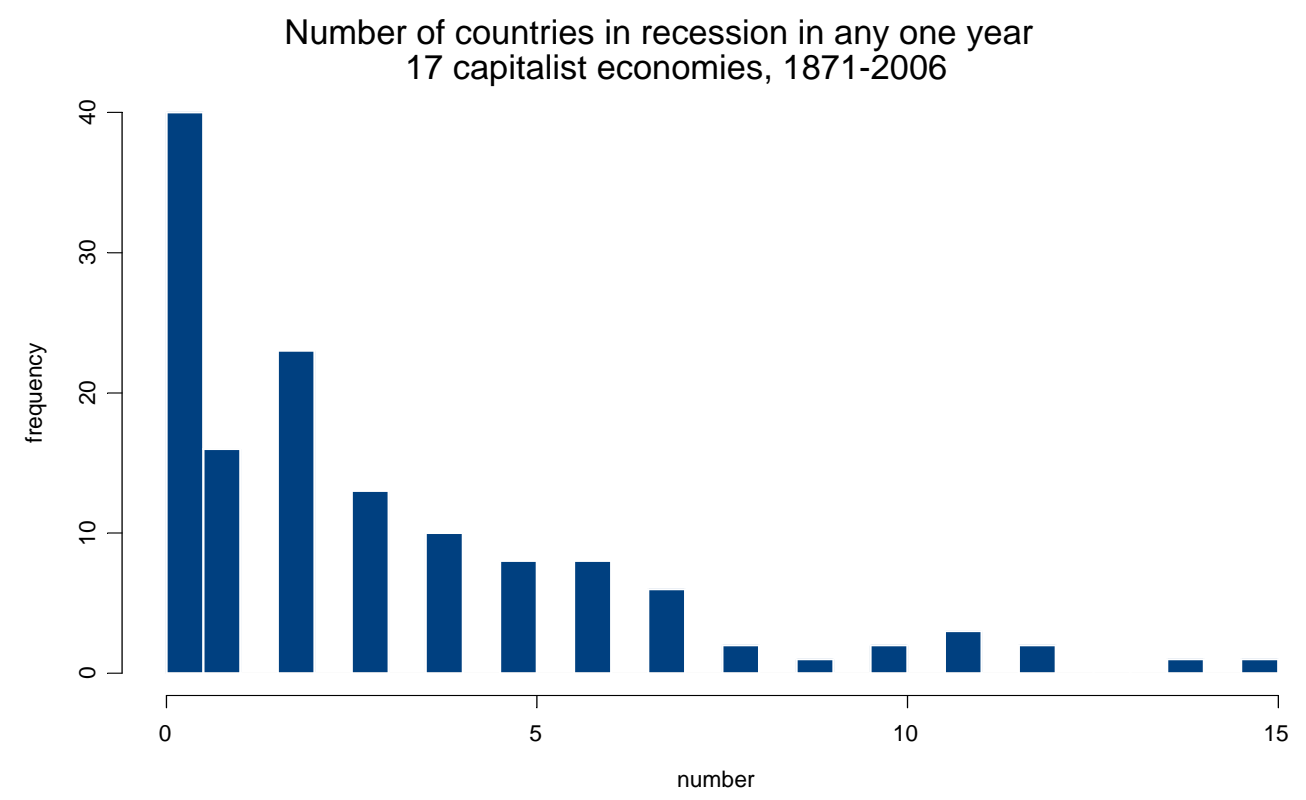

Figure 2 Number of countries in recession in any one year, 17 countries, 18712006 


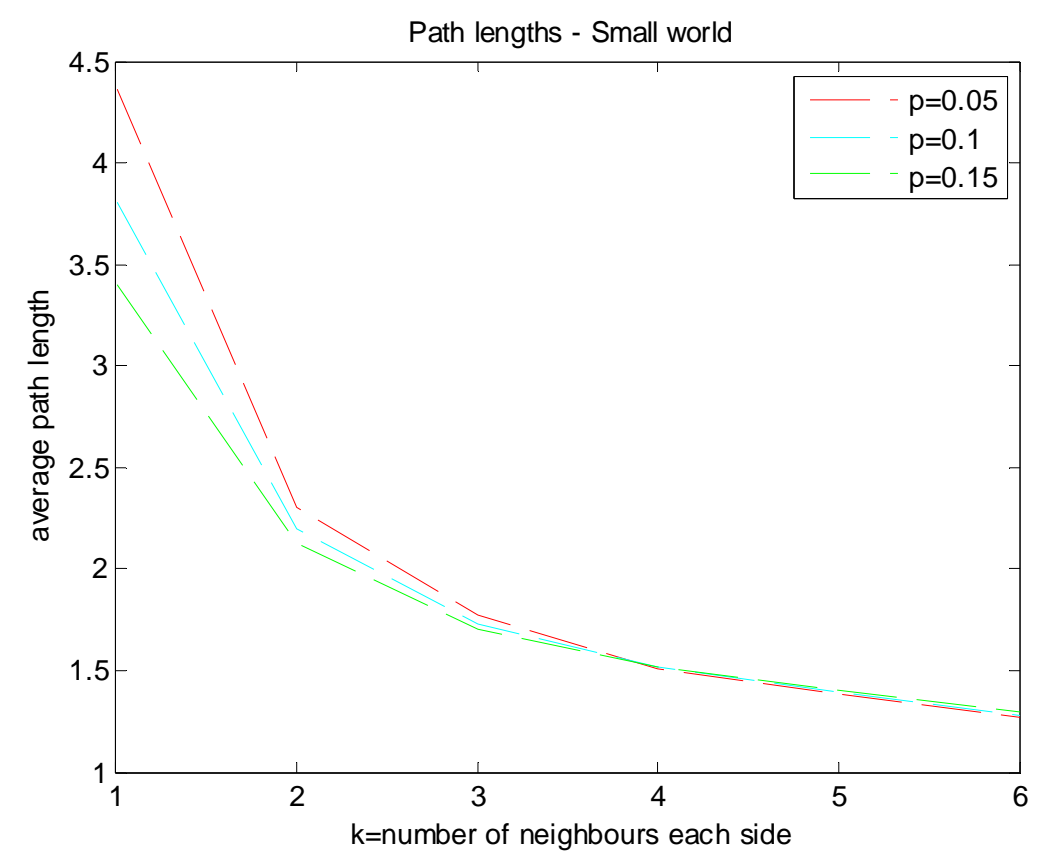

Figure 3 Average path length of small world network with 17 vertices, average over 500 separate realisations; rewiring replaces immediate neighbour connections; $p$ is the probability of re-wiring, $k$ is the number of immediate neighbours

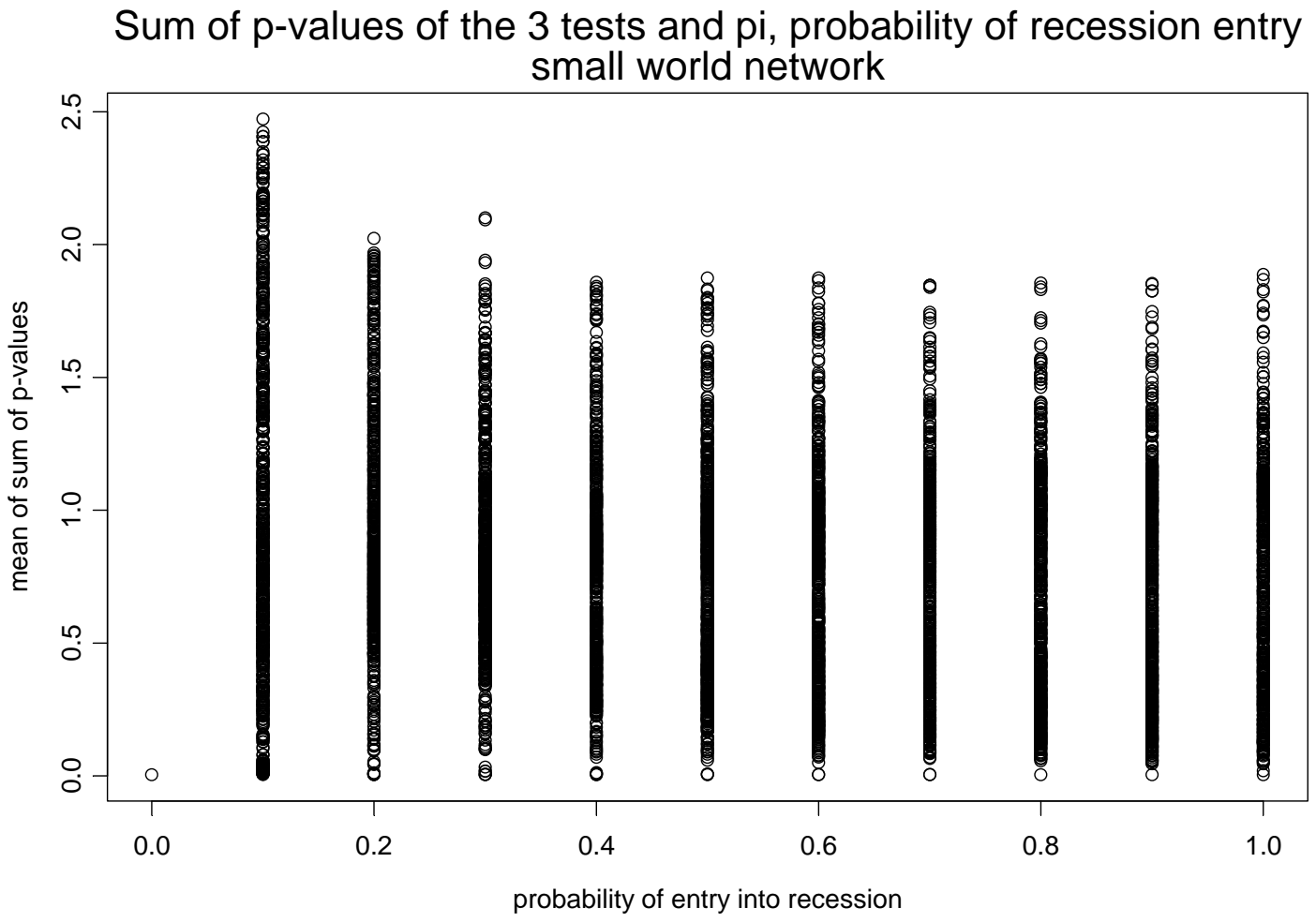


Figure 4a Average across all solutions of the sum of the p-values at which the null hypotheses is rejected that the distributions of the actual and model-generated data are the same, plotted for different values of the probability of a country entering a recession

Sum of $p$-values of the 3 tests and rho, probability of recession exit small world network

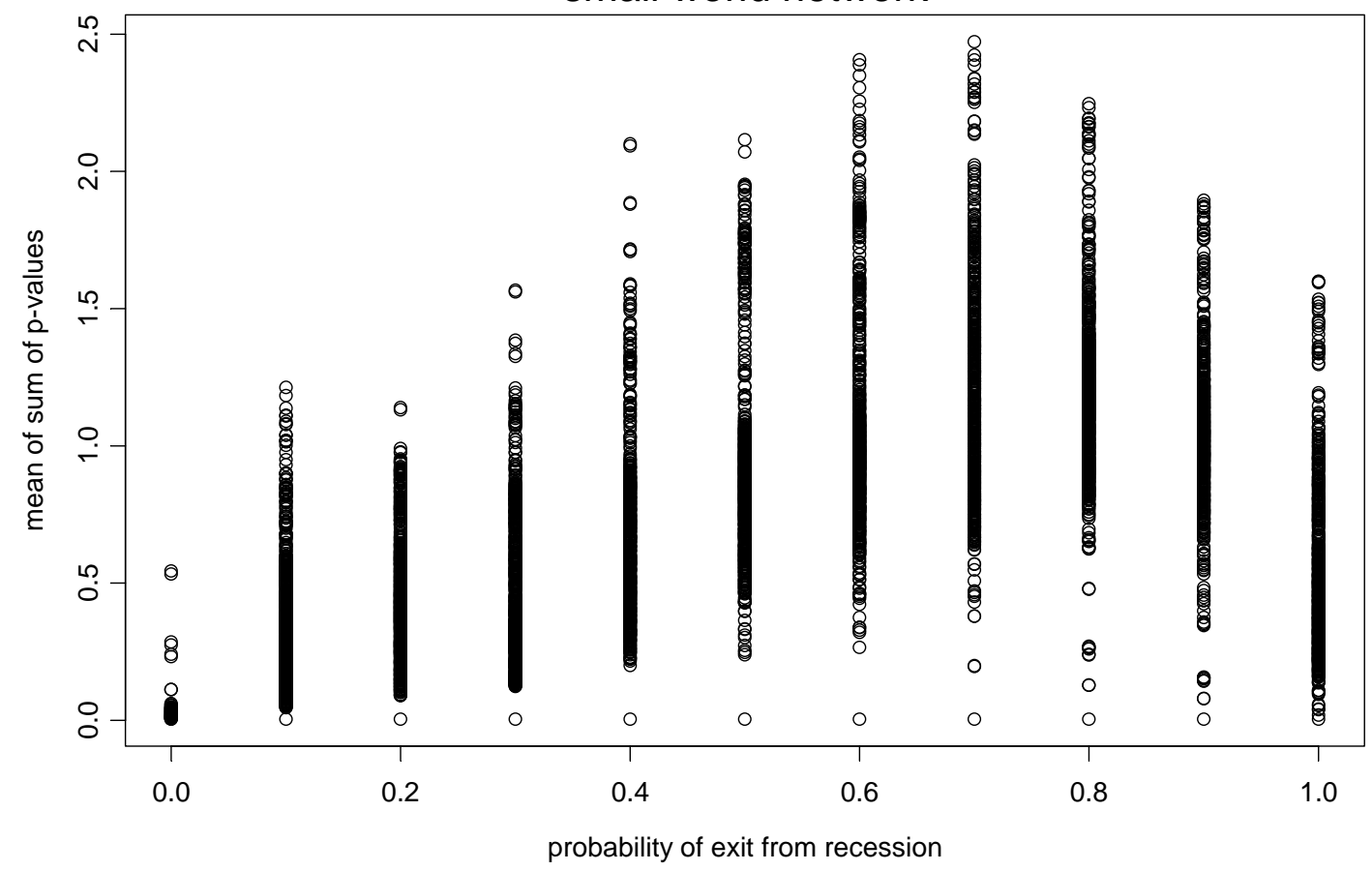

Figure 4b Average across all solutions of the sum of the p-values at which the null hypotheses is rejected that the distributions of the actual and model-generated data are the same, plotted for different values of the probability of a country exiting a recession 
Sum of $p$-values of the 3 tests and taumin small world network

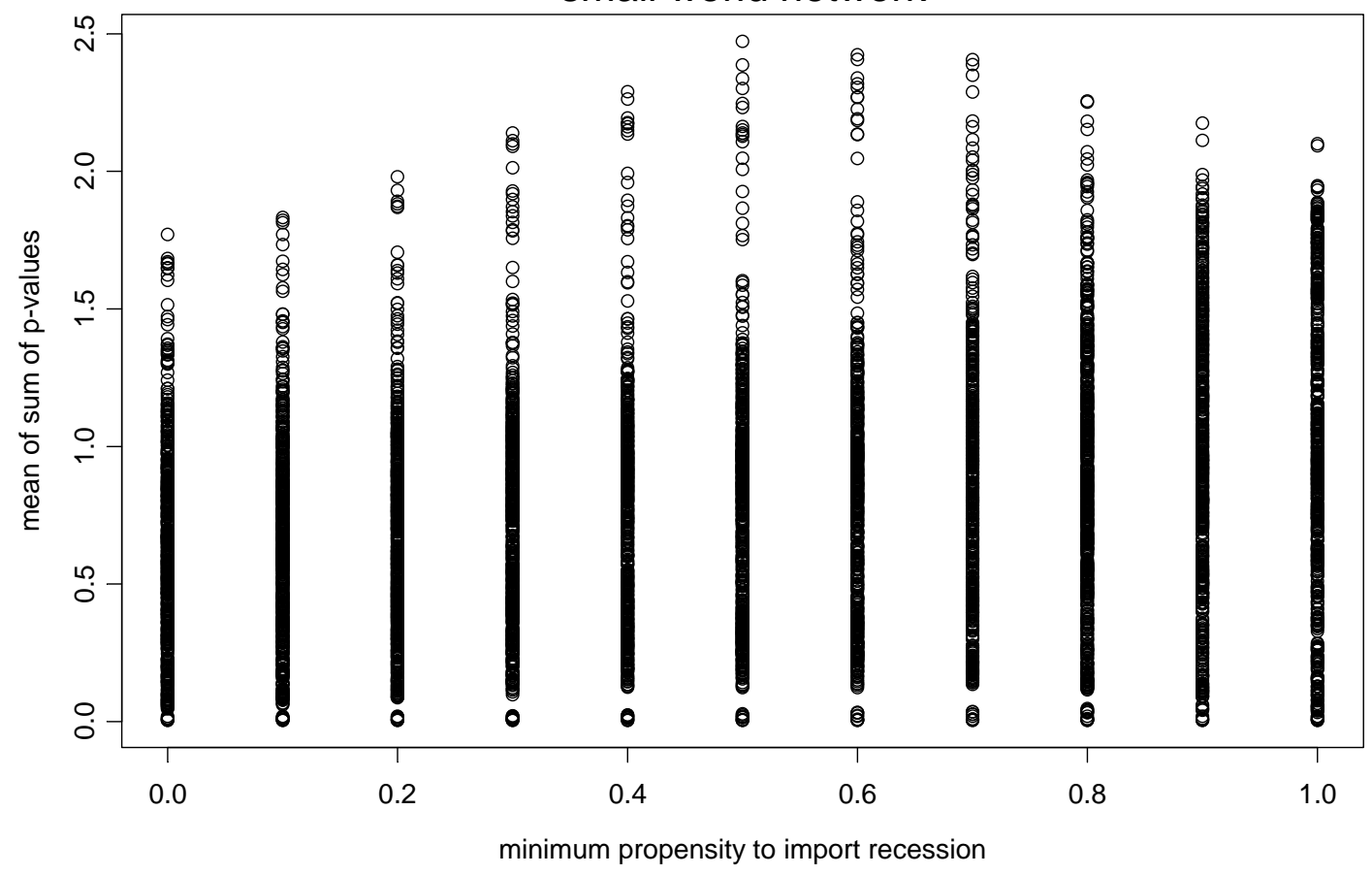

Figure 4c Average across all solutions of the sum of the p-values at which the null hypotheses is rejected that the distributions of the actual and model-generated data are the same, plotted for different values of the minimum probability of a country entering $a$ recession 